Bull. Austral. Math. Soc.

VOL. 65 (2002) [431-437]

\title{
TWO QUESTIONS ON SEMIGROUP LAWS
}

\author{
O. MACEDOŃSKA
}

B.H. Neumann recently proved some implications for semigroup laws in groups. This may help in the solution of a problem posed by G.M. Bergman in 1981.

\section{INTRODUCTION}

Let $G$ be a group, and $S \subseteq G$ be a subsemigroup generating $G$. It is clear that if $S$ is commutative, then $G$ is commutative. The following question is equivalent to the one posed by Bergman $[\mathbf{2}, \mathbf{3}]$.

Question 1 . Let $S$ generating $G$ satisfy a law. Must $G$ satisfy the same law?

For some laws the answer is positive $([\mathbf{9}, \mathbf{5}, \mathbf{8}, \mathbf{1}])$, however in general the question is open and in the opinion of Ivanov and Rips it has a negative answer. All semigroups we consider are cancellative.

QUESTION 2. Let a semigroup law $a=b$ imply a semigroup law $u=v$ in groups. Does the same implication hold in semigroups?

To show implication of laws in semigroups we can use only so-called positive endomorphisms, which map generators to positive words. It is shown in [8] (an example at the end of this paper), that all implications for positive laws of length $\leqslant 5$ which hold in groups, also are valid for semigroups. The fact that the law $x^{2} y^{2} x=y x^{3} y$ implies $x y=y x$ in semigroups (and hence in groups) is proved in [5, p.132].

We show the equivalence of the above Questions.

It is shown in [10], that the law $x^{s+t} y^{2} x^{t}=y x^{s+2 t} y, \operatorname{gcd}(s, t)=1$, implies $x y^{2} x=y x^{2} y$ in groups (which is equivalent to $[x, y, x]=1$ [12]). So if there exists a semigroup satisfying $x^{s+t} y^{2} x^{t}=y x^{s+2 t} y, \operatorname{gcd}(s, t)=1$, but not $x y^{2} x=y x^{2} y$, the desired counterexample for Question 1 would be found.

Let $a=a\left(x_{1}, \ldots, x_{n}\right), b=b\left(x_{1}, \ldots, x_{n}\right)$ be positive words. A semigroup law $a=b$ is called balanced if every $x_{i}$ has the same exponent sum in $a$ and $b$. The law is trivial if $a b^{-1}=1$ in $F$. The law is called cancelled if the first (and the last) letters in $a$ and $b$ are different.

Received 16th October, 2001

Copyright Clearance Centre, Inc. Serial-fee code: 0004-9727/02 \$A2.00+0.00. 
NotATION Let $F$ be a free group and $\mathcal{F} \ni 1$ be a free semigroup, both generated by $x_{1}, x_{2}, x_{3}, \ldots$ Words in $\mathcal{F}$ are called positive. We denote:

End $^{+}$- the set of positive endomorphisms which map $x_{i}$ to positive words,

$N_{w}$ - a normal End ${ }^{+}$-invariant closure of a word $w$ in $F$,

End - the set of all endomorphisms of the free group $F$,

$V_{w}$ - a fully invariant subgroup generated by a word $w \in F$,

$(u, v)^{\#}$ - the smallest cancellative congruence in $\mathcal{F}$ implying the law $u=v$.

A relatively free cancellative semigroup, defined by the law $u=v$ is isomorphic to $\mathcal{F} /(u, v)^{\#}[8]$.

We note that if $N_{w}$ contains a positive word, say $x^{2} y z^{4}$, then it contains $x^{7}$ and hence $x^{-1} \in x^{6} N_{w}$ implies $F=\mathcal{F} \bmod N_{w}$.

REMARK 1. Since each semigroup with a non-balanced law is a group, we have to consider only balanced non-trivial semigroup laws. Each such law implies a binary balanced and cancelled law $A(x, y)=B(x, y)([6])$.

\section{QUESTIONS AND RESUlts}

To formulate the above Questions in terms of normal subgroups we need

Lemma 1. A semigroup law $u=v$ implies $a=b$ in semigroups if and only if $N_{a b^{-1}} \subseteq N_{u v^{-1}}$. The implication holds in groups if and only if $V_{a b^{-1}} \subseteq V_{u v^{-1}}$.

ProOF: The law $u=v$ implies $a=b$ in semigroups if and only if the corresponding smallest congruences satisfy $(a, b)^{\#} \subseteq(u, v)^{\#}$. If we map $F \rightarrow F / N$, then $\mathcal{F}$ is mapped onto $\mathcal{F} / N^{\#}$, where $N^{\#}$ is a cancellative congruence in $\mathcal{F}$ defined as: $N^{\#}=\left\{(s, t) ; s t^{-1}\right.$ $\left.\in N \cap \mathcal{F F}^{-1}\right\}$. It is proved in [7, Theorem 2], that $N:=N_{u v^{-1}}$ is the smallest normal subgroup such that $N^{\#}=(u, v)^{\#}$. So we have

$$
(u, v)^{\#}=\left\{(s, t) ; s t^{-1} \in N_{u v^{-1}} \cap \mathcal{F F}^{-1}\right\} .
$$

Since $\mathcal{F} /(u, v)^{\#}$ is embeddable into a group $F / N_{u v^{-1}}$, and $N_{u v^{-1}}$ is the smallest normal subgroup with this property, it follows by $[4,12.3]$, that

$$
N_{u v^{-1}}=g p n\left(s t^{-1} ;(s, t) \in(u, v)^{\#}\right) .
$$

Hence by (1), (2): $(a, b)^{\#} \subseteq(u, v)^{\#}$ if and only if $N_{a b^{-1}} \subseteq N_{u v^{-1}}$, which gives the first statement of the Lemma. The second statement is known [11].

In terms of normal subgroups the above Questions are:

QUeSTION $1^{\prime}$. Does $N_{a b^{-1}}=V_{a b^{-1}}$ hold for each semigroup law $a=b$ ?

QUESTION 2'. Does $V_{a b^{-1}} \subseteq V_{u v^{-1}}$ imply $N_{a b^{-1}} \subseteq N_{u v^{-1}}$ for semigroup laws $a=b$ and $u=v$ ? 
We shall prove that for each semigroup law $a=b$ there exists a semigroup law $u=v$ such that the fully invariant closure of $a b^{-1}$ coincides with the End ${ }^{+}$-invariant normal closure of $u v^{-1}$. This will imply the equivalence of the Questions.

THEOREM. For every $n$-variable semigroup law $a=b$ there exists an $n+1$-variable semigroup law $u=v$ such that the equality $V_{a b^{-1}}=N_{u v^{-1}}$ holds.

COROLlary. Questions 1 and 2 are equivalent.

ProOF: We have to show that for each semigroup law $a=b$ the equality holds: $N_{a b^{-1}}=V_{a b^{-1}}$. Take $u=v$ as in the Theorem, then $V_{a b^{-1}} \stackrel{\mathrm{T}}{=} N_{u v^{-1}}$. By taking the fully invariant closure we get $V_{a b^{-1}}=V_{u v^{-1}}$. If Question 2 has a positive answer then we have $N_{a b^{-1}}=N_{u v^{-1}} \stackrel{\mathrm{T}}{=} V_{a b^{-1}}$, as required.

\section{Lemmas and Proof of the Theorem}

LEMMA 2. Let $A(x, y)=B(x, y)$ be a balanced and cancelled semigroup law such that the first letter in $A(x, y)$ is $x$. Then there exist $a_{i}=a_{i}(x, y), b_{i}=b_{i}(x, y) \in \mathcal{F}$, $i=1,2$, such that

(i) $x^{-1} y=a_{1} b_{1}^{-1} \cdot\left(A^{-1} B\right)^{b_{1}^{-1}}$,

(ii) $x y^{-1}=a_{2}^{-1} b_{2} \cdot\left(A B^{-1}\right)^{\varepsilon b_{2}}, \varepsilon= \pm 1$,

(iii) $F=\mathcal{F F}^{-1} N_{A B^{-1}}=\mathcal{F}^{-1} \mathcal{F} N_{A B^{-1}}$.

Proof: Since the law $A=B$ is cancelled, it can be written as $x \cdot a_{1}=y \cdot b_{1}$, which gives $A^{-1} B=a_{1}^{-1} x^{-1} y b_{1}$ and hence (i). The law $A=B$, (or $B=A$ ) can be written as $a_{2} \cdot x=b_{2} \cdot y$. In the first case $A B^{-1}=a_{2} x y^{-1} b_{2}$ gives $x y^{-1}=a_{2}^{-1} b_{2} \cdot\left(A B^{-1}\right)^{b_{2}}$. If $B=a_{2} \cdot x, A=b_{2} \cdot y$, then $x y^{-1}=a_{2}^{-1} b_{2} \cdot\left(A B^{-1}\right)^{-b_{2}}$, which gives (ii).

Since $A^{-1} B=\left(A B^{-1}\right)^{-B} \in N_{A B^{-1}}$, we get from (i), that $x^{-1} y \in \mathcal{F F}^{-1} \bmod N_{A B^{-1}}$, which holds under every substitution of elements from $\mathcal{F}$ for $x$ and $y$. Since every word in $F$ is a product of words in $\mathcal{F} \cup \mathcal{F}^{-1}$, we get $F=\mathcal{F} \mathcal{F}^{-1} N_{A B^{-1}}$. Similarly, from (ii) we get $F=\mathcal{F}^{-1} \mathcal{F} N_{A B^{-1}}$.

The following Lemma is well known in terms of a group of fractions and Ore conditions.

Lemma 3. Let $a=b$ be a nontrivial semigroup law, and $g_{1}, g_{2}, \ldots, g_{n}$ be elements in $F$. Then there exist elements $s_{1}, s_{2}, \ldots, s_{n}$ and $d$ in $\mathcal{F}$ such that $g_{i}=s_{i} d^{-1} \bmod N_{a b^{-1}}$.

Proof: By [6], the law $a=b$ implies a balanced and cancelled binary law $A=B$. Since $N_{A B^{-1}} \subseteq N_{a b^{-1}}$, the inclusions in Lemma 2 are valid $\bmod N_{a b^{-1}}$. Then by (iii) we have modulo $N_{a b^{-1}}: g_{i}=a_{i} b_{i}^{-1}$ for some $a_{i}, b_{i} \in \mathcal{F}$. For $n=2, g_{1}=a_{1} b_{1}^{-1}, g_{2}=a_{2} b_{2}^{-1}$. Also by (iii), there exist $c, d$ such that $b_{2}^{-1} b_{1}=c d^{-1}$. We introduce $r:=b_{1} d=b_{2} c$, then $g_{1}=a_{1} b_{1}^{-1}=a_{1} d d^{-1} b_{1}^{-1}=a_{1} d r^{-1}=: s r^{-1}, \quad g_{2}=a_{2} b_{2}^{-1}=a_{2} c c^{-1} b_{2}^{-1}=a_{2} c r^{-1}=$ : $t r^{-1}, s, t, r \in \mathcal{F}$. So, by repeating this step we can write $g_{1}, \ldots, g_{n}$ with a "common denominator" $\bmod N_{a b^{-1}}$ as required. 
To compare End ${ }^{+}$-invariant and End-invariant closures of words we make an observation that by positive endomorphisms we can map $x y^{-1}$ into any word $g \in F \bmod N_{a b^{-1}}$ if we write $g=s t^{-1}$ and map $x$ to $s$, and $y$ to $t$.

LEMMA 4. There exists an automorphism $\alpha \in$ Aut $F$ such that for any $w \in F$, $N_{w^{\alpha}}$ is fully invariant $\bmod N_{a b^{-1}}$, for any nontrivial $a b^{-1} \in \mathcal{F F}^{-1}$. That is $V_{w} \subseteq$ $N_{w^{\alpha}} N_{a b^{-1}}$.

ProOF: Let $w=w\left(x_{1}, \ldots, x_{n}\right)$. We take $\alpha \in$ Aut $F$ which maps $x_{i} \rightarrow x_{i} x_{n+1}^{-1}$, $i=1, \ldots, n$ and leaves $x_{i}, i>n$, fixed. It is enough to show that for any $g_{1}, \ldots, g_{n}$ in $F, w\left(g_{1}, \ldots, g_{n}\right) \in N_{w^{\alpha}} N_{a b^{-1}}$. By Lemma 3, we write $g_{i}=s_{i} d^{-1} \bmod N_{a b^{-1}}$ and define $\nu \in$ End $^{+}$by $x_{i}^{\nu}=s_{i}, i \leqslant n$, and $x_{n+1}^{\nu}=d$. Then modulo $N_{a b^{-1}}$ we have $\left(x_{i} x_{n+1}^{-1}\right)^{\nu}=g_{i}$ and $w\left(g_{1}, \ldots, g_{n}\right)=w\left(x_{1} x_{n+1}^{-1}, \ldots, x_{n} x_{n+1}^{-1}\right)^{\nu}=\left(w\left(x_{1}, \ldots, x_{n}\right)^{\alpha}\right)^{\nu} \in N_{w^{\alpha}}^{\nu} \subseteq N_{w^{\alpha}}$, as required.

COROLLARY 1. For a nontrivial semigroup law $a=b$ the following equality holds:

$$
V_{a b^{-1}}=N_{\left(a b^{-1}\right)^{a}}
$$

ProOF: We have $a b^{-1} \in N_{\left(a b^{-1}\right)^{\alpha}}^{\alpha^{-1}}$. Since $\alpha^{-1}$ is in End ${ }^{+}$, then $N_{\left(a b^{-1}\right)^{\alpha}}^{\alpha^{-1}} \subseteq N_{\left(a b^{-1}\right)^{a}}$ and hence $a b^{-1} \in N_{\left(a b^{-1}\right)^{\alpha}}$, which gives

$$
N_{a b^{-1}} \subseteq N_{\left(a b^{-1}\right)^{\alpha}}
$$

By Lemma 4 for $w:=a b^{-1}$, by (3), and since End ${ }^{+} \subseteq$ End, we have:

$$
V_{a b^{-1}} \subseteq N_{\left(a b^{-1}\right)^{a}} N_{a b^{-1}}=N_{\left(a b^{-1}\right)^{\alpha}} \subseteq V_{a b^{-1}}
$$

which implies $V_{a b^{-1}}=N_{\left(a b^{-1}\right)^{\alpha}}$.

We denote by $\delta$ the endomorphism which maps $x_{n+1} \rightarrow 1$ and leaves other generators fixed, then $\delta \in$ End $^{+}$. As above, $\alpha \in$ Aut $F$ maps $x_{i} \rightarrow x_{i} x_{n+1}^{-1}, i=1, \ldots, n$ and leaves $x_{i}, i>n$, fixed.

LEMMA 5. Let $a=b$ be a nontrivial semigroup law, and $\mathcal{F}_{n+1}$ be a free subsemigroup generated by $x_{1}, \ldots, x_{n+1}$. Then for any positive word $p\left(x_{1}, \ldots, x_{n}\right)$, there exist positive words $u_{i}=u_{i}\left(x_{1}, \ldots, x_{n+1}\right), v_{i}=v_{i}\left(x_{1}, \ldots, x_{n+1}\right), i=1,2$, such that $p^{\alpha}=u_{1} v_{1}^{-1}=u_{2}^{-1} v_{2} \bmod \left(N_{a b^{-1}} \cap \operatorname{Ker} \delta\right)$.

Proof: We show first that for any words $c, q \in \mathcal{F}_{n+1}$ the following inclusions hold:

$$
\begin{aligned}
& c x_{n+1}^{-1} \in \mathcal{F}_{n+1}^{-1} \mathcal{F}_{n+1} \bmod \left(N_{a b^{-1}} \cap \operatorname{Ker} \delta\right), \\
& x_{n+1}^{-1} q \in \mathcal{F}_{n+1} \mathcal{F}_{n+1}^{-1} \bmod \left(N_{a b^{-1}} \cap \operatorname{Ker} \delta\right) .
\end{aligned}
$$

The law $a=b$ implies the balanced and cancelled binary law $A=B$, so it is enough to prove the inclusions for the law $A(x, y)=B(x, y)$. 
If we apply $\delta$ to the balanced equality $A\left(c, x_{n+1}\right)=B\left(c, x_{n+1}\right)$, it becomes trivial, and hence the word $A B^{-1}\left(c, x_{n+1}\right)$ is in $\operatorname{Ker} \delta$. Similarly we get $A^{-1} B\left(x_{n+1}, q\right) \in \operatorname{Ker} \delta$. We put now $c, x_{n+1}$, for $x, y$, in (ii) (Lemma 2) to get (*), and then put $x_{n+1}, q$, in (i) (Lemma 2) to get $(* *)$.

We continue the proof modulo $\left(N_{a b^{-1}} \cap \operatorname{Ker} \delta\right)$. To show that:

$$
p\left(x_{1} x_{n+1}^{-1}, \ldots, x_{n} x_{n+1}^{-1}\right) \in \mathcal{F}_{n+1} \mathcal{F}_{n+1}^{-1},
$$

and

$$
p\left(x_{1} x_{n+1}^{-1}, \ldots, x_{n} x_{n+1}^{-1}\right) \in \mathcal{F}_{n+1}^{-1} \mathcal{F}_{n+1},
$$

we use induction on the length $|p|=m$. Let $p\left(x_{1}, \ldots, x_{n}\right)=c_{m} c_{m-1} \ldots c_{2} c_{1}, c_{i}$ $\in\left\{x_{1}, \ldots, x_{n}\right\}$, then $p^{\alpha}=c_{m} x_{n+1}^{-1} c_{m-1} x_{n+1}^{-1} \ldots c_{2} x_{n+1}^{-1} c_{1} x_{n+1}^{-1}$. For $m=1, p^{\alpha}=c x_{n+1}^{-1}$ $\in \mathcal{F}_{n+1} \mathcal{F}_{n+1}^{-1}$ and by $(*), p^{\alpha}=c x_{n+1}^{-1} \in \mathcal{F}_{n+1}^{-1} \mathcal{F}_{n+1}$.

Let $|p|=m$, then $p=c_{m} c_{m-1} \ldots c_{2} c_{1}$ and by inductive assumption $p^{\alpha}=c_{m} x_{n+1}^{-1}$. $q r^{-1}$. Then by $(* *)$, there exist $s, t \in \mathcal{F}_{n+1}$, such that $x_{n+1}^{-1} q=s t^{-1}$ and hence $p^{\alpha}=c_{m}\left(x_{n+1}^{-1} q\right) r^{-1}=c_{m}\left(s t^{-1}\right) r^{-1}=\left(c_{m} s\right)(r t)^{-1} \in \mathcal{F}_{n+1} \mathcal{F}_{n+1}^{-1}$.

Again for $|p|=m$, we get by assumption $p^{\alpha}=r^{-1} s \cdot c_{1} x_{n+1}^{-1}=r^{-1}\left(s c_{1}\right) x_{n+1}^{-1}$. By (*) for $s c_{1}$ instead of $c$, there exist $t, u \in \mathcal{F}_{n+1}$, such that $s c_{1} x_{n+1}^{-1}=t^{-1} u$. Then $p^{\alpha}=r^{-1}\left(s c_{1}\right) x_{n+1}^{-1}=r^{-1} t^{-1} u=(t r)^{-1} u \in \mathcal{F}_{n+1}^{-1} \mathcal{F}_{n+1}$ as required.

\section{Proof of The Theorem}

We have to show that for every nontrivial $n$-variable semigroup law $a=b$ there exists an $n+1$-variable semigroup law $u=v$ such that $V_{a b^{-1}}=N_{u v^{-1}}$.

By Lemma 5 for the words $a=a\left(x_{1}, \ldots, x_{n}\right)$ and $b=b\left(x_{1}, \ldots, x_{n}\right)$ we get respectively:

$$
a^{\alpha}=u_{1} v_{1}^{-1} \bmod \left(N_{a b^{-1}} \cap \operatorname{Ker} \delta\right)
$$

and

$$
b^{\alpha}=u_{2}^{-1} v_{2} \bmod \left(N_{a b^{-1}} \cap \operatorname{Ker} \delta\right)
$$

Then

$$
\left(a b^{-1}\right)^{\alpha}=u_{1} v_{1}^{-1} v_{2}^{-1} u_{2}=u_{2}^{-1}\left(u_{2} u_{1}\right)\left(v_{2} v_{1}\right)^{-1} u_{2} \bmod \left(N_{a b^{-1}} \cap \operatorname{Ker} \delta\right) .
$$

We denote $u:=u_{2} u_{1}, v:=v_{2} v_{1}$, then

$$
\left(a b^{-1}\right)^{a}=\left(u v^{-1}\right)^{u_{2}} \bmod \left(N_{a b^{-1}} \cap \operatorname{Ker} \delta\right)
$$

This implies:

$$
N_{\left(a b^{-1}\right)^{\alpha}} \subseteq N_{u v^{-1}} N_{a b^{-1}}
$$

and

$$
N_{u v^{-1}} \subseteq N_{\left(a b^{-1}\right)^{\alpha}} N_{a b^{-1}}
$$


To prove the equality

$$
N_{\left(a b^{-1}\right)^{\alpha}}=N_{u v^{-1}}
$$

we apply $\delta$ to (4). Since $\alpha \delta$ is the identity map on $x_{i}, i \leqslant n$, and $\delta$ is in End ${ }^{+}$, we have that $a b^{-1}=\left(a b^{-1}\right)^{\alpha \delta}$ is conjugate to $\left(u v^{-1}\right)^{\delta} \in N_{u v^{-1}}^{\delta} \subseteq N_{u v^{-1}}$. This implies $N_{a b^{-1}} \subseteq N_{u v^{-1}}$ which, together with (5) gives $N_{\left(a b^{-1}\right)^{\alpha}} \subseteq N_{u v^{-1}}$. Since by (3), $N_{a b^{-1}} \subseteq N_{\left(a b^{-1}\right)^{\alpha}}$, it follows from (6), that $N_{u v^{-1}} \subseteq N_{\left(a b^{-1}\right)^{\alpha}}$, and hence (7) holds.

Now, since by Corollary $1, V_{a b^{-1}}=N_{\left(a b^{-1}\right)^{\alpha}}$, we have by (7), the required equality $V_{a b^{-1}}=N_{u v^{-1}}$.

\section{EXAMPLE OF IMPLICATIONS IN SEMIGROUPS}

[8] The law $(x y)^{2}=(y x)^{2}$ implies $x y^{2}=y^{2} x$ for groups because we can apply the automorphism $\alpha: x \rightarrow x, y \rightarrow x^{-1} y$. For semigroups we can not use this automorphism. To prove that $(x y)^{2}=(y x)^{2}$ implies $x y^{2}=y^{2} x$ for semigroups we show first that $(x y)^{2}$ $=(y x)^{2}$ implies:

$$
\begin{array}{lll}
\text { (i) }(y x)^{2} y=y(y x)^{2}, & \text { (use the word } \left.y(x y)^{2}\right), \\
\text { (ii) } x\left((y x)^{2} y\right)^{2}=\left((y x)^{2} y\right)^{2} x, & \text { (use }(i)^{\alpha}, x^{\alpha}=x y x^{2}, y^{\alpha}=y \text {; and } x \leftrightarrow y \text { ) } \\
\text { (iii) }\left((y x)^{2} y\right)^{2}=(y x)^{4} y^{2}, & \text { (use } \left.\left((y x)^{2} y\right)\left((x y)^{2} y\right)\right), \\
\text { (iv) }(y x)^{4}=(x y)^{4} . &
\end{array}
$$

Then for some word $p$ we start with $p \cdot x y^{2}$ and by using (i)-(iv) obtain $p \cdot y^{2} x$, which by cancellation, implies required $x y^{2}=y^{2} x$. Namely, for $p=(x y)^{4}$ we have

$$
\begin{aligned}
& p x y^{2}=(x y)^{4} x y^{2}=x(y x)^{2} \underline{(y x)^{2} y} y \stackrel{(\mathrm{i})}{=} x(y x)^{2} \underline{y(y x)^{2}} y \\
& =x\left((y x)^{2} y\right)^{2} \stackrel{(\text { ii) }}{=}\left((y x)^{2} y\right)^{2} x \stackrel{(\text { iii) }}{=}(y x)^{4} y^{2} x \stackrel{\text { (iv) }}{=}(x y)^{4} y^{2} x=p y^{2} x,
\end{aligned}
$$

which gives $p x y^{2}=p y^{2} x$ and hence $x y^{2}=y^{2} x$ as required.

\section{REFERENCES}

[1] R.G. Burns, O. Macedońska and Y. Medvedev,, 'Groups satisfying semigroup laws, and nilpotent-by-Burnside varieties', J. Algebra 195 (1997), 510-525.

[2] G.M. Bergman, 'Hyperidentities of groups and semigroups', Aequationes Math. 23 (1981), 50-65.

[3] G.M. Bergman, 'Questions in algebra', (preprint, Berkeley, U.C. 1986).

[4] A.H. Clifford and G.B. Preston, The algebraic theory of semigroups, Math. Surveys 7 (Amer. Math. Soc., Providence R.I., 1964).

[5] J. Krempa and O. Macedońska, 'On identities of cancellative semigroups', Contemp. Mathem. 131 (1992), 125-133.

[6] J. Lewin and T. Lewin, 'Semigroup laws in varieties of soluble groups', Proc. Cambridge Philos. Soc. 65 (1969), 1-9. 
[7] O. Macedońska, 'On cancellative congruences for semigroups', Zeszyty Nauk. Politech. Slaskiej Mat.-Fiz. 84 (1999), 171-176.

[8] O. Macedońska and M. Żabka, 'On equivalence of semigroup identities', Math. Scand. 88 (2001), 161-181.

[9] A.I. Malcev, 'Nilpotent semigroups', Ivanov. Gos. Ped. Inst. Uc. Zap. Fiz. Mat. Nauki 4 (1953), 107-111.

[10] B.H. Neumann, 'Some semigroup laws in groups', Canad. Math. Bull. 44 (2001), 93-96.

[11] H. Neumann, Varieties of groups (Springer-Verlag,, Berlin, Heidelberg, New York, 1967).

[12] A.I. Shirshov, 'On some positively defined varieties of groups', Siberian Math. J. (1959), 165-178.

Institute of Mathematics

Silesian Technical University

ul. Kaszubska 23

44-100 Gliwice

Poland

e-mail: olga@zeus.polsl.gliwice.pl 